\title{
Linear Algebra and its Applications
}

\section{Imbedding conditions for normal matrices}

\author{
João Filipe Queiró*, António Leal Duarte \\ CMUC, Department of Mathematics, University of Coimbra, 3001-454 Coimbra, Portugal
}

\section{ARTICLE INFO}

\section{Article history:}

Received 1 March 2008

Accepted 15 August 2008

Available online 8 October 2008

Submitted by V. Mehrmann

To Thomas Laffey, on his 65th birthday.

AMS classification:

$15 \mathrm{~A} 29$

$15 \mathrm{~A} 42$

$15 \mathrm{~A} 57$

\section{Keywords:}

Normal matrix

Principal submatrix

Compression

Eigenvalues

Interlacing

Lexicographic order

\section{A B S T R A C T}

When can an $(n-k) \times(n-k)$ normal matrix $B$ be imbedded in an $n \times n$ normal matrix $A$ ? This question was studied for the first time 50 years ago by Ky Fan and Gordon Pall, who gave the complete answer in the case $k=1$. Since then, a few authors obtained additional results. In this note, we show how an approach inspired by the Hermitian case can throw some light on the problem.

(c) 2008 Elsevier Inc. All rights reserved.

\section{Introduction}

Given complex matrices $A n \times n$, and $B(n-k) \times(n-k)$, we say $B$ is imbeddable in $A$ - or, equivalently, $B$ is a compression of $A-$ if $B$ is a principal submatrix of $U^{*} A U$ for some $U n \times n$ unitary.

Fifty years ago, Fan and Pall [3] posed, and solved, the following problem: if $A$ and $B$ are Hermitian, when is $B$ imbeddable in $A$ ? The answer-a necessary and sufficient condition involving the eigenvalues $\alpha_{1} \geqslant \ldots \geqslant \alpha_{n}$ of $A$ and $\beta_{1} \geqslant \ldots \geqslant \beta_{n-k}$ of $B$-is given by the well-known interlacing inequalities $\alpha_{j} \geqslant \beta_{j} \geqslant \alpha_{j+k}, j=1, \ldots, n-k$.

\footnotetext{
* Corresponding author.

E-mail addresses: jfqueiro@mat.uc.pt (J.F. Queiró), leal@mat.uc.pt (A.L. Duarte).
} 
In the same paper, Fan and Pall asked the same question for normal matrices. They solved the problem in the $k=1$ case. The answer is that imbeddability happens essentially only (apart from a rotation and a translation) in the Hermitian case.

Theorem 1.1 [3]. Let $A$ be an $n \times n$ normal matrix with eigenvalues $\alpha_{1}, \ldots, \alpha_{n}, B$ an $(n-1) \times(n-1)$ normal matrix with eigenvalues $\beta_{1}, \ldots, \beta_{n-1}$. Renumber the eigenvalues so that $\alpha_{j}=\beta_{j-1}, j=q+1, \ldots, n$ and $\alpha_{1}, \ldots, \alpha_{q}$ are each distinct from $\beta_{1}, \ldots, \beta_{q-1}$. Then $B$ is imbeddable in $A$ if and only if the $2 q-1$ points $\alpha_{1}, \ldots, \alpha_{q}, \beta_{1}, \ldots, \beta_{q-1}$ are collinear and the $\beta$ 's separate the $\alpha$ 's on that line.

The sufficiency part in this result is trivial (because of the Hermitian result). The real content of the result is the necessity part.

In the Hermitian case, only the the $n-1$ case is needed for the reciprocal in the general $n-k$ case: we just need to insert intermediate sequences of eigenvalues, for a chain of matrices. This is not so in the normal case, as shown by the following example in [3]. Take

$$
A=\operatorname{diag}(0,1, i, 1+i) \text { and } B=\frac{1}{10} \operatorname{diag}(5+8 i, 5+2 i) .
$$

Then $B$ is imbeddable in $A$ but there does not exist a $3 \times 3$ normal matrix $C$ such that $B$ is imbeddable in $C$ and $C$ is imbeddable in $A$.

Fan and Pall's interesting result for normal matrices in the $k=1$ case was not followed by the analysis of what happens for larger $k$. We know about only two later papers about this kind of question.

In 1984, Carlson and Marques de Sá [1] proved the following result on the same problem.

Theorem 1.2. Let $A$ be an $n \times n$ normal matrix with (nonzero) eigenvalues $\alpha_{1}, \ldots, \alpha_{n}$, satisfying $\gamma+\pi>$ $\arg \alpha_{1} \geqslant \cdots \geqslant \arg \alpha_{n} \geqslant \gamma$ for some $\gamma \geqslant 0$. Let $B$ be an $(n-k) \times(n-k)$ normal principal submatrix of $A$. Then the eigenvalues $\beta_{1}, \ldots, \beta_{n-k}$ of $B$ may be ordered so that $\gamma+\pi>\arg \beta_{1} \geqslant \cdots \geqslant \arg \beta_{n-k} \geqslant \gamma$ and

$$
\arg \alpha_{j} \geqslant \arg \beta_{j} \geqslant \arg \alpha_{j+k}, \quad j=1, \ldots, n-k .
$$

Later, in a work published in 1998 [5], Ikramov and Elsner studied the slightly different question of finding conditions under which an $(n-k) \times(n-k)$ normal matrix $B$ can be-nontrivially-dilated to an $n \times n$ normal matrix $A$, with special attention to the $k=1$ and $k=2$ cases.

There is some literature concerning principal (not necessarily normal) submatrices of normal matrices. We mention here one of the earliest papers on that other problem, by Thompson [9], and two of the most recent, one by Malamud [7], and one by Savchenko [8].

In this paper, we explore the idea of using the lexicographic order in $\mathbb{C}$ to mimic the Hermitian case (where interlacing is the complete answer), and we find that this throws some light on the normal imbeddability problem.

\section{Lexicographic orders in $\mathbb{C}$}

The lexicographic order is characterized by the positive cone

$$
H=\{a+i b: a>0 \text {, or } a=0 \text { and } b>0\} .
$$

For real $\theta$, define $\leqslant_{\theta}$ as the total order with positive cone $\mathrm{e}^{\mathrm{i} \theta} H$ (so the lexicographic order is $\leqslant_{0}$ ). These orders are total (i.e., all numbers are comparable) and compatible with addition and with multiplication by positive reals (i.e., $\alpha \leqslant_{\theta} \beta \Rightarrow \alpha+\gamma \leqslant{ }_{\theta} \beta+\gamma$ and, if $\rho$ is positive, $\alpha \leqslant_{\theta} \beta \Rightarrow \rho \alpha \leqslant_{\theta} \rho \beta$ ). They are the only orders in $\mathbb{C}$ having those properties. We have of course

$$
\alpha \leqslant_{\theta} \beta \Longleftrightarrow \mathrm{e}^{-\mathrm{i} \theta} \alpha \leqslant_{0} \mathrm{e}^{-\mathrm{i} \theta} \beta
$$

The condition $\alpha \geqslant_{\theta} \beta$ means that, when we sweep the plane with parallel lines orthogonal to the $\arg \theta$ direction, intersecting that direction before going to the $\arg (\theta+\pi)$ one, we find $\alpha$ before we find 
$\beta$ (in case of a tie, $\alpha$ is found to the right of $\beta$ ). The numbering of elements in decreasing sequences of course depends on $\theta$.

\section{Min-max for normal matrices}

Let $\theta \in \mathbb{R}$ be arbitrary. Take $A n \times n$ normal, and let $\alpha_{1}, \ldots, \alpha_{n}$ be the eigenvalues of $A$, ordered so that $\alpha_{1} \geqslant_{\theta} \ldots \geqslant_{\theta} \alpha_{n}$. Let $v_{1}, \ldots, v_{n}$ be corresponding orthonormal eigenvectors of $A$. For $j=1, \ldots, n$, denote by $E_{j}$ and $E_{j}^{\prime}$ the subspaces spanned by $v_{1}, \ldots, v_{j}$ and $v_{j}, \ldots, v_{n}$, respectively.

Theorem 3.1. For $j=1, \ldots, n$ we have

$$
\alpha_{j}=\min _{x \in E_{j},\|x\|=1} x^{*} A x=\max _{x \in E_{j}^{\prime},\|x\|=1} x^{*} A x .
$$

Here and in similar circumstances max and min are used in the $\leqslant_{\theta}$ sense.

The proof is the same as for the Hermitian case (see e.g. [2]). Analogously, we can prove that

$$
\alpha_{j}=\max _{\operatorname{dim} E=j} \min _{x \in E,\|x\|=1} x^{*} A x=\min _{\operatorname{dim} E=n-j+1} \max _{x \in E,\|x\|=1} x^{*} A x .
$$

Actually, all of the known extremal characterization of eigenvalues of Hermitian matrices by means of the associated quadratic forms carry over to normal matrices using the $\theta$-lexicographic orders. For example, we have the following generalization of Theorem 3.1:

$$
\alpha_{j_{1}}+\cdots+\alpha_{j_{r}}=\min _{L \in \Omega_{j}(E)} \operatorname{tr}\left(A_{\mid L}\right)=\max _{L \in \Omega_{J^{\prime}}\left(E^{\prime}\right)} \operatorname{tr}\left(A_{\mid L}\right),
$$

where $J=\left(j_{1}, \ldots, j_{r}\right), J^{\prime}=\left(n-j_{r}+1, \ldots, n-j_{1}+1\right), E=\left(E_{1}, \ldots, E_{n}\right)$, and $E^{\prime}=\left(E_{n}^{\prime}, \ldots, E_{1}^{\prime}\right)$, the $\Omega$ denote the Schubert varieties associated to the respective sequences of indices and subspaces, and $\operatorname{tr}\left(A_{\mid L}\right)$ is the Rayleigh trace of $A$ with respect to $L$ (see [4] for the Hermitian case). This characterization can be applied, by analyzing intersection of Schubert varieties, to obtaining inequalities for the eigenvalues of a sum of two normal matrices with given eigenvalues, if this sum is itself normal. In the Hermitian case (where the sum is always Hermitian, of course), the family of inequalities thus obtained is the complete description of the possible spectra of the sum [6].

Before returning to the imbeddability problem, we obtain a well-known result with a simple application of the characterization of the $\theta$-first eigenvalue of $A$ as the $\theta$-maximum of $x^{*} A x$ on the unit sphere.

Theorem 3.2. The numerical range $W(A)=\left\{x^{*} A x:\|x\|=1\right\}$ of a normal matrix $A$ is the convex hull of its eigenvalues.

Proof. Any straight line moving parallel to itself in the plane must touch $W(A)$ first at an eigenvalue of $A$.

\section{Interlacing for normal matrices}

Theorem 4.1. Let $\theta$ be arbitrary. Let $A$ be an $n \times n$ normal matrix with eigenvalues $\alpha_{1} \geqslant \geqslant_{\theta} \cdots \geqslant_{\theta} \alpha_{n}$. If $B$ is a principal $(n-k) \times(n-k)$ normal submatrix of $A$ with eigenvalues $\beta_{1} \geqslant_{\theta} \cdots \geqslant_{\theta} \beta_{n-k}$, we have

$$
\alpha_{j} \geqslant_{\theta} \beta_{j} \geqslant_{\theta} \alpha_{j+k}, \quad j=1, \ldots, n-k \text {. }
$$

Proof. The proof is the same as in the Hermitian case [2], using min-max.

Of course, the eigenvalues of a normal matrix are the complex numbers whose real and imaginary parts are the eigenvalues of the Hermitian and skew-Hermitian parts of the matrix. But interlacing of real and imaginary parts does not imply lexicographic interlacing, as simple examples show. The 
interlacing theorem for normal matrices can be obtained from the Hermitian case, but the above proof is as good as any other, and in any case our interest is in the result itself, which we will apply immediately.

\section{Corollary 1. Theorem 1.1 above.}

Proof. The sufficiency is trivial, since we are essentially in the Hermitian case, as mentioned before. As to the necessity, take $q \alpha$ 's distinct from $q-1 \beta$ 's as in Theorem 1.1. From Theorem 4.1 we know that, for every $\theta$, the $\beta$ 's separate the $\alpha$ 's with respect to the $\geqslant_{\theta}$ order. We claim this implies that between any two $\alpha$ 's there must be a $\beta$. Indeed, given two $\alpha$ 's, if we sweep the plane with parallel lines very slightly rotated with respect to the line through those two points, the interlacing condition for that rotated direction guarantees that there must be a $\beta$ between the two $\alpha$ 's. A simple counting argument now shows that the $q \alpha$ 's and the $q-1 \beta$ 's must be collinear.

Corollary 2. Theorem 1.2 above.

Proof. Assume without loss of generality that $\gamma=0$, i.e. the $\alpha$ 's satisfy $\pi>\arg \alpha_{1} \geqslant \ldots \geqslant \arg \alpha_{n} \geqslant 0$ (this is just for graphical reasons). The $\beta$ 's then lie also on the upper half-plane, as they belong to $W(B)$ and trivially $W(B) \subseteq W(A)$. Order them so that $\arg \beta_{1} \geqslant \cdots \geqslant \arg \beta_{n-k}$.

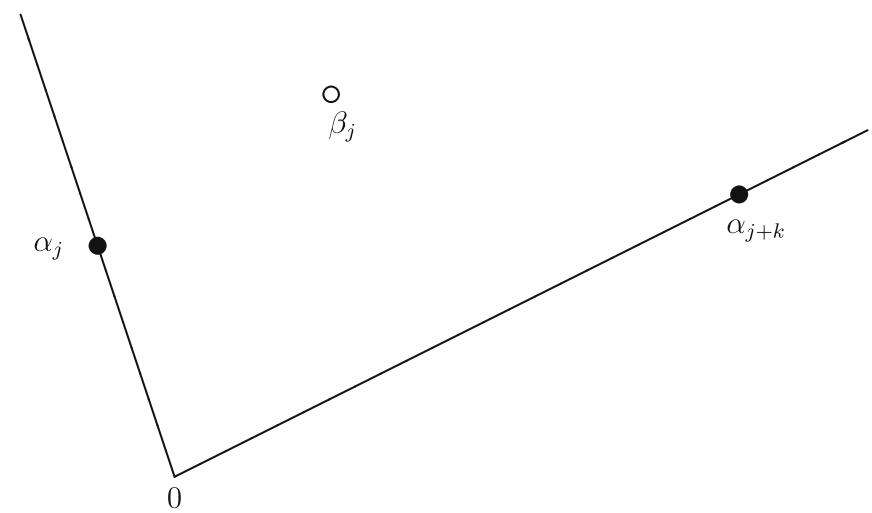

If we sweep the plane left to right with lines parallel to the line through 0 and $\alpha_{j}$, then clearly $\alpha_{j}$ is the $j$ th $\alpha$ we find. By Theorem 4.1, $\beta_{j}$ must lie to the right of the line through 0 and $\alpha_{j}$. Similarly, if we sweep the plane right to left with lines parallel to the line through 0 and $\alpha_{j+k}$, then $\alpha_{j+k}$ is the $(j+k)$ th $\alpha$ we find. By Theorem 4.1, $\beta_{j}$ must lie to the left of the line through 0 and $\alpha_{j+k}$.

A remark made during the proof of Corollary 1 can be generalized, with the same argument.

Theorem 4.2. Let $A$ be an $n \times n$ normal matrix with eigenvalues $\alpha^{\prime}$, having a principal $(n-k) \times(n-k)$ normal submatrix $B$ with eigenvalues $\beta$ 's. If $k+1$ of the $\alpha$ 's are collinear, there must be $a$ in the segment defined by those $k+1 \alpha$ 's.

Proof. If we sweep the plane with parallel lines very slightly rotated with respect to the line through the $k+1 \alpha$ 's, the interlacing condition for that rotated direction guarantees that there must be a $\beta$ in the segment defined by those $k+1$ points.

The same reasoning can go even further, to show that, if $k+p$ of the $\alpha$ 's are collinear, there must be $p \beta$ 's in the segment defined by those $k+p \alpha$ 's, one between the first and the $(p+1)$ th $\alpha$ (counting from one extreme point of the segment to the other), one between the second and the $(p+2)$ th, and so on. 


\section{Two open questions}

Two questions now emerge:

(1) What restrictions does the $\geqslant_{\theta}$ interlacing theorem impose on the eigenvalue configurations? (recall the $k=1$ case).

(2) What additional conditions must be added to the $\geqslant_{\theta}$ interlacing theorem (all $\theta$ ) to obtain the complete answer for the imbedding problem in the general $n-k$ case?

The first question is purely geometric, with no relation to matrices. Let us make some remarks on it. For $n=3$ and $k=2$, the interlacing condition is just $\alpha_{1} \geqslant_{\theta} \beta_{1} \geqslant_{\theta} \alpha_{3}($ all $\theta)$, and it is easy to see that this is equivalent to the single $\beta$ lying in the triangle defined by the three $\alpha$ 's. It is also easy to see that this is the complete solution for the imbedding problem.

For $n>3$, this can be generalized by the following result.

Theorem 5.1. Given $n \alpha$ 's and $n-2 \beta$ 's, suppose that they satisfy the interlacing condition in Theorem 4.1 for all $\theta$ (and $k=2$ ). Take one $\alpha$ on the boundary of the convex hull of the $\alpha$ 's. Draw half-lines from this point to all the other $n-1 \alpha$ 's. Then in each of the $n-2$ sectors so formed there is a $\beta$.

Proof. Of course, since $W(B) \subseteq W(A)$, we already know the $\beta$ 's belong to the convex hull of the $\alpha$ 's.

Name the chosen $\alpha$ as $\alpha_{1}$, and number the others, starting from $\alpha_{1}$, so that the half-lines from $\alpha_{1}$ rotate consecutively (clockwise, say) from one side of $\alpha_{1}$ to the other.

Denote by $\triangleleft\left(\alpha_{i}, \alpha_{j}\right)$ the sector formed by the half lines from $\alpha_{1}$ to $\alpha_{i}$ and $\alpha_{j}$, including those half lines. There must a $\beta$ in the sector $\triangleleft\left(\alpha_{2}, \alpha_{3}\right)$, because of interlacing with respect to lines parallel to the segment $\left[\alpha_{1}, \alpha_{3}\right]$.

Next, there must a $\beta$ in the sector $\triangleleft\left(\alpha_{3}, \alpha_{4}\right)$ : this is because of interlacing with respect to lines parallel to the segment $\left[\alpha_{1}, \alpha_{4}\right]$, which forces two $\beta$ 's to lie in the convex hull of $\alpha_{1}, \alpha_{2}, \alpha_{3}, \alpha_{4}$ : one of them we already know is in the sector $\triangleleft\left(\alpha_{2}, \alpha_{3}\right)$, and the other cannot be in (the interior of) the same sector, as that would mean that, with respect respect to lines parallel to the segment $\left[\alpha_{1}, \alpha_{3}\right]$, the second $\beta$ would come (strictly) before the second $\alpha$.

Similarly, there must a $\beta$ in the sector $\triangleleft\left(\alpha_{j+1}, \alpha_{j+2}\right)$, for all $j$.

An interesting consequence follows immediately in the case of convex independence of the $\alpha$ 's.

Corollary 3. Given $n \alpha$ 's and $n-2 \beta$ 's, suppose that they satisfy the interlacing condition in Theorem 4.1 for all $\theta$, and $k=2$. If the $\alpha$ 's are convexly independent (i.e., none of them belongs to the convex hull of the others), then in any triangle with vertices on $\alpha$ 's there is a $\beta$.

Simple examples, already with $n=4$, show that the conclusion of Corollary 3 may fail in the nonindependent case.

Theorem 5.1 can be generalized, with the same reasoning, for $k>2$. Numbering the $\alpha$ 's as in the proof of that theorem, the statement then is that, in each of the $n-k$ sectors $\triangleleft\left(\alpha_{j}, \alpha_{j+k-1}\right), j=2, \ldots, n-$ $k+1$, there is a $\beta$.

Returning to the case $k=2$, we conjecture that the triangle condition mentioned in Corollary 3 is in fact necessary and sufficient for the $\theta$ interlacing conditions when the $\alpha$ 's are independent. This seems an interesting geometric problem. When $n=4$ (independent case), the condition for interlacing is that the two $\beta$ 's lie on opposite sectors with respect to the diagonals of the quadrilateral defined by the $\alpha$ 's (recall the Fan-Pall example).

\section{Acknowledgments}

We thank John Holbrook for interesting conversations about the last section. 


\section{References}

[1] D. Carlson, E. Marques de Sá, Generalized minimax and interlacing theorems, Linear Multilinear Algebra 15 (1984) 77-103.

[2] R. Courant, D. Hilbert, Methods of Mathematical Physics, vol. I, Interscience Publishers, New York, 1953.

[3] Ky Fan, G. Pall, Imbedding conditions for Hermitian and normal matrices, Canad. J. Math. 9 (1957) 298-304.

[4] J. Hersch, B. Zwahlen, Évaluations par défaut pour une somme quelconque de valeurs propres $\gamma_{k}$ d'un opérateur $C=A+B$ à l'aide de valeurs propres $\alpha_{i}$ de $A$ et $\beta_{j}$ de $B$, C.R. Acad. Sci. Paris 254 (1962) 1559-1561.

[5] K.D. Ikramov, L. Elsner, On normal matrices with normal principal submatrices, J. Math. Sci. 89 (1998) 1631-1651.

[6] A. Klyachko, Stable bundles, representation theory and hermitian operators, Selecta Math. 4 (1998) 419-445.

[7] S.M. Malamud, Inverse spectral problem for normal matrices and the Gauss-Lucas theorem, Trans. AMS 357 (2005) 40434064.

[8] S.V. Savchenko, Normal matrices and their principal submatrices of co-order one, Linear Algebra Appl. 419 (2006) $556-568$.

[9] R.C. Thompson, Principal submatrices of normal and Hermitian matrices, Illinois J. Math. 10 (1966) 296-308. 